\title{
DIVERSITY, EXCLUSION AND RISK, AS SECOND-LANGUAGE LEARNERS OF IMMIGRANT PARENTS ACQUIRE FIRST-TIME LITERACY IN ENGLISH
}

\author{
Norma Nel \& Ms Elizabeth Claire Gien Snelgar \\ University of South Africa
}

\begin{abstract}
Research has shown that acquisition of literacy skills and the ultimate realisation of literacy, which involves comprehension of the written text, require more than the ability to decode individual words. This study provides a synopsis of current research on the topics of globalisation, the resultant cultural incompatibility in the classroom, emergent literacy, vocabulary development, reading, and reading comprehension. As such, it offers a discussion of a comparative study of limited English-proficient (LEP)/English language learners (ELLs) acquiring first-time literacy, with the attendant vocabulary deficits and lack of ageappropriate decoding skills. Quantitative and qualitative approaches were combined to examine the differences between reading skills, comprehension, and vocabulary when a learner born of foreign parents acquires first-time literacy in a language other than his or her home language. Statistical techniques were used to analyse and interpret the research results. Analysis of the study results isolates and specifies an at-risk educational minority through the identification of a hidden comprehension deficit (HCD).
\end{abstract}

\section{Keywords}

English language learner; limited English proficiency; hidden comprehension deficit; rapid automatic naming; phonemic awareness; comprehension deficit; second language

\section{INTRODUCTION}

English language learners (ELL's) acquiring first-time literacy in a second language (SL) are an emerging phenomenon in Grades 1, 2 and 3 in most English-speaking countries today. For the purposes of this study, these learners are also deemed limited English proficient (LEP). The challenge for teachers in the English-speaking inclusive classroom is to prepare both ELLs and monolingual learners for the acquisition of the required literacy skills. In view of the difficulties associated with teaching literacy skills to these diverse groups of learners, we focused on a resultant phenomenon of globalisation, namely the ELL, referred to in this research as an at-risk educational minority, based on their LEP. We also attempted to identify this at-risk educational minority linguistically, culturally and socially. In addition, the hidden comprehension deficit (HCD) manifested in this group of learners is identified and described. For clarification and ease of reference, the source and definition follow, as well as a brief explanatory note:

The hidden comprehension deficit has been conceptualised by the author. HCD refers to a comprehension deficit, prevalent in 
learners acquiring first-time literacy, in a language that is not spoken at home. The comprehension deficit is masked by ageappropriate reading, rapid automatic naming (RAN) and phonemic awareness skills (PA) and therefore, is often undetected. (Gien Snelgar, 2010:9)

The study stems not from a deficit model but from the model of improving on strengths and identifying differences and, in so doing, abolishing exclusion in inclusive classrooms. Robertson (2005:714) described inclusive education as a 'welcoming environment for all students, not by ignoring gender, race, disability [language proficiency] or sexual identities, but by recognizing and validating difference'. For ongoing research, vigorous rhetoric, comparative analysis and, ultimately, incorporation of findings into pedagogical practices, it is essential to highlight differences in order to improve upon existing classroom and teaching realities. In addition, for the purpose of this study, ESL speakers are deemed to have proficiency and emergent literacy skills in the mother tongue, of which there are over 200 (excluding the aboriginal languages) in British Columbia (Statistics Canada, 2006b:1). These strengths are acknowledged but not addressed in this research. The overarching motivation of this research (see endnote) and subsequent article is to initiate and facilitate the specialised care of the needs of this heterogeneous population, namely the ESL acquiring literacy within an inclusive school system. Inclusion, at present, is an evolutionary ideology, with an attendant school system that is in constant flux -- a system that requires tremendous flexibility in its organisation and curriculum planning, particularly as the effects of globalisation become more apparent within the school system. More importantly, this system urgently requires an in-depth investigation and insight into the mechanics of the ESL learners' linguistic diversity, in order to address their specific needs at a practical, hands-on level. This calls for the diversification of teaching strategies, teaching methods and materials.

The study was focused on Grades 1, 2 and 3 learners in British Columbia, Canada (the age distribution varied between 6 and 9 years, with a median age of 7 years and 4 months), where English is legislated as the primary language for teaching, learning, examinations, textbooks and school governance (Protocol for Agreements for Ministry Language Education and Second Language 2009-2010 to 2012-2013). However, 1 in every 5 people in British Columbia belongs to a visible minority (HELP, 2001:36), whose home language is other than English. The residents of urban districts, primarily the populous Lower Mainland of British Columbia, constitute a large number of ELLs. More specifically, roughly more than half the population of Richmond, Vancouver and Burnaby (municipalities within Metro Vancouver which have a large ethnic heritage linked to China and South Asia) are members of a visible minority (HELP, 2001:46). The ELL, born in Canada of immigrant parents, who acquires first-time literacy is characterised by differential and deficient English proficiency, vocabulary and comprehension levels and is therefore deemed at risk of lacking the required English literacy skills. Fundamental to the acquisition of literacy skills and positive academic outcomes is language proficiency and adequate vocabulary and comprehension levels. Cain and Oakhill (2007:31) noted increasing evidence of a common basis of basic language skills which underpins the development of written as well as spoken language comprehension. Research describing the difficulties of elementary ELLs is a comprehensive and welldocumented field. However, limited research is available on acquiring first-time literacy in a second language. This article reports on the differences between reading, comprehension and vocabulary when learners born of foreign parents acquire first-time literacy in Grades 1, 2 and 3 in a language other than their home languages. These differences were identified by means 
of a combination of qualitative and quantitative research approaches. Statistical techniques were used to analyse and interpret the research results. Analysis also isolates and specifies an at-risk educational minority specified through the identification of a hidden comprehension deficit (HCD).

\section{THEORETICAL FRAMEWORK}

Over the past two decades, global migration has increased and continues to increase significantly, bringing about rapid transformation of the affected societies and the education systems that function within these societies. In 2007, 70.2\% of immigrants (to Canada) or foreign-born residents reported a mother tongue other than English or French (Statistics Canada, 2006a:18). Given the role of schools as 'major mediators of life's opportunities' (CCL, 2008:1) and Canada's resolute constitutional commitment to equality and multiculturalism, it is essential to develop and integrate appropriate support for these ELLs to ensure positive outcomes in the short term and long term. The large number and wide variety of foreign residents in British Columbia has increased the necessity for vigorous investigation of ELLs and their varied outcomes (Garner, 2008; Gunderson, 2007).

Language development is the quintessential ingredient for the acquisition of literacy skills. For this reason, language defines the broad framework used in this study of monolingual learners and ELLs, specifically with regard to their language proficiency, vocabulary and comprehension levels. Trower and Robinson (cited in Barnhill, 2001:260) posited that social behaviour is the most central and important characteristic of human beings. One of the many consequences of globalisation and inclusion is the disconnect that becomes evident when familial socialisation and language use are not compatible with the societal and classroom socialisation norms. This results in a 'cultural mismatch' (Hakuta, 2001:87), which creates a vulnerability for the ELL acquiring first-time literacy. ELLs experience a distinct disadvantage in addressing the challenges of the social milieu found in the classroom because of their divergent socio-linguistic and cultural backgrounds, emergent literacy skills and difficulties with first-time literacy acquisition.

Emergent literacy skills are acquired in the home environment. For the ELL in British Columbia, this takes place in a home where English is not the first language and often not spoken at all by parents or caregivers. The ELL's first language (FL) (mother tongue spoken in the home) and emergent literacy skills develop and, in addition, are richly imbued with the cultural and social interaction in the home. This, however, is no substitute for the ritualistic shared reading of Mother Goose, the attendant awareness of phonemes, the rhyming and alliteration embedded in 'Hickory, Dickory, Dock, the mouse ran up the clock' and other rhymes serve as latent building blocks for sound awareness, which 'teach the young ear' (Wolf, 2007:99) and ensure seamless literacy acquisition in English, while demonstrating the symbolic nature of print and the sounds represented. The ELL's ritualistic shared reading of early childhood, and thus exposure to print and sound awareness, takes place in the FL spoken at home. In the English alphabet, a systematic relationship occurs between the letters of the alphabet and the phonemes of the language, whereas Mandarin or Cantonese make use of a character system, each character representing a word. Furthermore, emergent literacy skills are greatly influenced through social and cultural interaction, where young learners acquire literacy from everyday experiences with more literate adults (Morrow, 2001:253). Clearly, different aspects of the home literacy environment affect the emergent literacy exposure, classroom compatibility and development of each learner differently. 
Effective literacy acquisition is dependent on the learners' attendant oral proficiency, vocabulary levels, cultural compatibility and emergent literacy skills. The development of the learner's phonological ear is also crucial to literacy acquisition -- more specifically, the recognition of speech units that form different words. These speech units have been 'recognised to play [as playing] a causal role in the acquisition of literacy (Goswami, cited in Neumann \& Dickenson, 2003:111). Moreover, identifying the printed word, ascribing meaning to the words, and building a lexicon are critical to the acquisition of literacy skills. Muter et al. (2004:667) elaborated on the need to distinguish word recognition skills from comprehension skills, as comprehension skills pertain to varying, complex underlying language skills. Language comprehension linked to proficient vocabulary levels addresses the challenges of the visible minority and the identification of the HCD.

When an ELL starts school, the learner has a divergent, but equally rich, complex language and vocabulary framework structured on the mother tongue or home language. There is evidence that variations in learners' environments are associated with marked differences in language and vocabulary development (Hart \& Risley, 2003:4). This is crucial when considering the fundamental anomalies in language, vocabulary, paralinguistics and semiotics of ELLs and monolingual learners in Grades 1,2 and 3 and the application of these variations to first-time literacy acquisition. Mitchell and Myles (2004:55) substantiated the described difficulties by referring to the 'messy and fragmented' input of language being problematic in grasping abstract concepts based on limited examples of language. In other words, the language to which the ELL is exposed in the classroom and the inadequate exposure to English in the home environment are not supportive of adequate vocabulary development and ultimate literacy acquisition.

The challenge of second language acquisition, vocabulary and comprehension for the ELL and, more specifically, for those who enter Grades 1, 2 and 3 with limited, if any, proficiency in English, is an almost insurmountable task. In sum, the monolingual learner has a 5-to 6year head start on language development, which begins with sensitivity to the mother tongue in utero and as a neonate (Kamiloff \& Kamilloff-Smith, 2001:17), while ELLs are just starting off. ELLs face an enormous, but perhaps not insoluble, problem of vocabulary acquisition in a compressed timeframe. In addition, ELLs' vocabulary learning and emergent literacy skills and exposure to sophisticated vocabulary, language rich in syntax and semantic contexts, alliteration in rhyme, phoneme recognition and manipulation thereof are in deficit. The Grade 1 ELL who is not buoyed up by supportive language scaffolding is at high risk of incurring poor acquisition of literacy skills since he or she is embedded in a foreign and paralysing linguistic, cultural and social environment, which is not conducive to successful acquisition of literacy skills. These learners have a 5- to 6-year handicap and are further beset by LEP, extensive vocabulary deficits, and therefore comprehension deficits, when trying to realise literacy in its fullest sense.

Comprehension is dependent on vocabulary development, cultural compatibility and exposure. However, it is the combined skill of the reading of the word or text, and extracting meaning from the word or text, which is critical to reading success. Reading for meaning (comprehension) is the ability to deduce comprehensive understanding and to extract meaning from the text as opposed to an understanding of individual words or sentences. Therefore, vocabulary is a major determinant of comprehension. As text passages become more complex, poor vocabulary levels limit meaning, creating a phenomenon termed the 'white 
space' (Hakuta et al., 2000). In other words, comprehension and meaning diminish and the gap between meaning and decoding becomes greater.

ELLs' deficits in emergent literacy skills, vocabulary, language proficiency, class room compatibility and comprehension relate to and support difficulties in extracting word meaning from the written text. These deficits support the notion of the development of a hidden comprehension deficit (HCD), as posited by Gien Snelgar (2010), in ELLs acquiring firsttime literacy in English.

\section{RESEARCH METHODOLOGY}

The research was guided and supported by the theoretical foundation and conceptual framework in the literature review. The research objective called for a multidisciplinary enquiry encompassing both a quantitative, non-experimental, comparative mode and a qualitative, interactive, micro-ethnographic mode of enquiry, which inform the research design. A multidisciplinary enquiry enables different disciplines 'to do their best' (Kanbar, 2002:477-486) in creating a richer and more useful dialogue and synthesis of results. The qualitative mode of enquiry, an interview/questionnaire, substantiates the linguistic background of the ELL group. Insight into the parents' and learners' social reality underpins the quantitative data, a reading and vocabulary assessment.

\section{THE RESEARCH QUESTION}

Can statistically significant differences be established between age-appropriate reading, comprehension and vocabulary levels in ELLs (whose home language is other than English), during first-time literacy acquisition, when English is the language used in school and the classroom, compared to monolingual learners, whose home language is English and who are acquiring first-time literacy in a classroom/school where English is used as the only medium of tuition and which ELL's attend as well?

\section{HYPOTHESIS}

The null and alternative hypotheses, which can be formulated from the main research question, could be formally stated as follows:

Null hypothesis $\mathrm{H}_{0 \mathrm{~m}}$ :

There are no statistically significant differences in literacy performance of ELLs (whose home language is other than English) and monolingual learners (whose home language is English), evaluated by age-appropriate reading, comprehension and vocabulary levels, when learners are exposed to first-time literacy acquisition in a school/classroom environment where English is the language of instruction.

Alternative hypothesis $\mathrm{H}_{1 \mathrm{~m}}$ :

There are statistically significant differences in literacy performance of ELLs (whose home language is other than English) and monolingual learners (whose home language is English) evaluated by age-appropriate reading, comprehension and vocabulary levels, when learners are exposed to first-time literacy acquisition in a school/classroom environment where English is the language of instruction. 
The deficit was investigated in ELLs of immigrant parents whose home language is other than English and who acquired first-time literacy exposure in English in Grades 1-3.

\section{SUB-HYPOTHESIS}

The most effective way of evaluating the main hypothesis would be to split the main hypothesis into the two components of literacy ability and evaluate achievement on each aspect separately. To this effect, sub-hypotheses were formulated as follows:

Age-appropriate reading null hypothesis, $\mathrm{H}_{\mathrm{O} 1}$ :

There is no statistically significant difference in literacy performance of ELLs (whose home language is other than English) and monolingual learners (whose home language is English), evaluated by age-appropriate reading, when learners are exposed to first-time literacy acquisition in a school and classroom environment where English is the language of instruction.

Age-appropriate reading alternative hypothesis $\mathrm{H}_{11}$ :

There is a statistically significant difference in literacy performance of ELLs (whose home language is other than English) and monolingual learners (whose home language is English), evaluated by age-appropriate reading, when learners are exposed to first-time literacy acquisition in a school and classroom environment where English is the language of instruction.

For performance on vocabulary levels, the null hypothesis $\mathrm{H}_{02}$ states that

There is no statistically significant difference in literacy performance of ELLs (whose home language is other than English) and monolingual learners (whose home language is English), evaluated on vocabulary levels, when learners are exposed to first-time literacy acquisition in a school and classroom environment where English is the language of instruction.

For performance on vocabulary levels, the alternative hypothesis $\mathrm{H}_{12}$ is

There is a statistically significant difference in literacy performance of ELLs (whose home language is other than English) and monolingual learners (whose home language is English), evaluated on vocabulary levels, when learners are exposed to first-time literacy acquisition in a school and classroom environment where English is the language of instruction.

\section{THE QUALITATIVE STUDY}

The main objective of the qualitative data collected in the study was to assist in profiling the HCD and at-risk communities of ELLs with regard to their cultural and social backgrounds. To achieve this objective, a questionnaire/interview probing language attributes was used to link the cultural and social perspectives. The language attributes are specifically targeted in this study. The responses from parents to the qualitative interview/questionnaire were recorded as response categories and summarised in frequency distribution tables. In addition, a structured observation instrument was used for the observation of the learners' behavioural attributes prior to, during, and after the reading analysis. The data were analysed and the results serve as a source of relevant information in identifying, describing and exploring an atrisk and a not-at-risk HCD educational minority, which complements the quantitative study. 
The qualitative study is based on data collected from $18(n=18)$ English SL learners and 19 $(n=19)$ English learners (monolinguals) in Grades 1, 2 and 3. The first group represents the experimental group, henceforth referred to as ELLs, and the second group, the comparison group, is henceforth referred to as English monolingual learners. The concepts experimental group and ELL as well as comparison group and English monolingual learners are used interchangeably in the study. The second group are English monolingual learners born of English-speaking Canadian parents, while the first group consists of Canadian-born learners of immigrant parents, who speak a language other than English at home. These learners jointly represent the purposeful sampling units or respondents sampled from the target population and are regarded as English learners in Grades 1 to 3 acquiring first-time literacy. The respondents were selected in the Surrey, Richmond and Burnaby areas of Vancouver. These areas are dominated by an Asian immigrant population with Mandarin or Cantonese as their first language spoken exclusively at home by learners, siblings and parents. Sociocultural, language competence, including current use of L1 and L2, literary ability (reading and writing), biographical details, history, inclusive of ELL tutoring for parent and learner data, were collected from parents of the ELLs (using the services of a translator) at a Vancouver college. In addition, a structured observation instrument was used to measure the ELLs' behavioural attributes prior to, during, and after the reading analysis of monolingual learners at the local community centre, daycare centre and local library.

The interview/questionnaire consisted of 30 questions, and responses to each question were directly recorded by the researcher as one of a number of response-category options. The information collected was summarised in a composite frequency table to describe and profile the cultural and social backgrounds of the participating English monolingual learners and ELLs, according to biographical attributes probed. Questions 3, 6, 18.1, 18.2, 19, 20, 21 and 25 specifically described language attributes of the sampled learner population and were regarded as crucial indicators of an at-risk educational minority through the identification of potential HCD in further analyses. The seven crucial attributes are listed in the condensed composite one-way frequency table, Table 1 presented below, illustrating the biographical trends underlying the condensed frequency distribution.

Table 1: Linguistic background of ELLs

Table 1

Linguistic background of ELL: Frequency distribution of the eight most crucial language attributes addressed in the structured parental interview/questionnaire

\begin{tabular}{|c|l|c|c|c|c|}
\hline \multirow{2}{*}{$\begin{array}{c}\text { Question } \\
\text { No }\end{array}$} & \multicolumn{1}{|c|}{ Question } & \multicolumn{2}{|c|}{$\begin{array}{c}\text { Monolingual } \\
\left(\mathrm{n}_{1}=19\right)\end{array}$} & \multicolumn{2}{c|}{$\begin{array}{c}\text { ELL } \\
\left(\mathrm{n}_{2}=18\right)\end{array}$} \\
\cline { 3 - 6 } & \multicolumn{1}{|c|}{$\begin{array}{c}\text { Frequency } \\
\text { ('yes' } \\
\text { response })\end{array}$} & $\%$ & $\begin{array}{c}\text { Frequency } \\
\text { ('yes' } \\
\text { response) }\end{array}$ & $\%$ \\
\hline Q 3 & Was the learner born in Canada? & 19 & 100 & 18 & 100 \\
\hline Q 6 & $\begin{array}{l}\text { Have the parents resided in Canada their } \\
\text { entire lives? }\end{array}$ & 19 & 100 & 0 & 0 \\
\hline Q 18.1 & $\begin{array}{l}\text { Do you speak English as mother tongue } \\
\text { at home? }\end{array}$ & 19 & 100 & 0 & 0 \\
\hline Q 18.2 & $\begin{array}{l}\text { Do you speak Mandarin/ Cantonese as } \\
\text { mother tongue at home? }\end{array}$ & 0 & 0 & 18 & 100 \\
\hline Q 19 & $\begin{array}{l}\text { Can you read/write in your mother } \\
\text { tongue? }\end{array}$ & 19 & 100 & 18 & 100 \\
\hline
\end{tabular}




\begin{tabular}{|c|l|c|c|c|c|}
\hline Q 20 & $\begin{array}{l}\text { Do you as a parent receive English } \\
\text { tuition? }\end{array}$ & 0 & 0 & 18 & 100 \\
\hline Q 21 & $\begin{array}{l}\text { Are you, the parent, able to read and write } \\
\text { in English? }\end{array}$ & 19 & 100 & 9 & 50 \\
\hline Q 25 & $\begin{array}{l}\text { Is your child exposed to English via } \\
\text { friends? }\end{array}$ & 19 & 100 & 4 & 22 \\
\hline
\end{tabular}

Furthermore, the frequency distributions between English monolingual learners and ELLs on linguistic attributes are illustrated in a bar graph plot presented in Figure 1.

\section{FIGURE 1: (BAR GRAPH TAKEN FROM TABLE 1)}

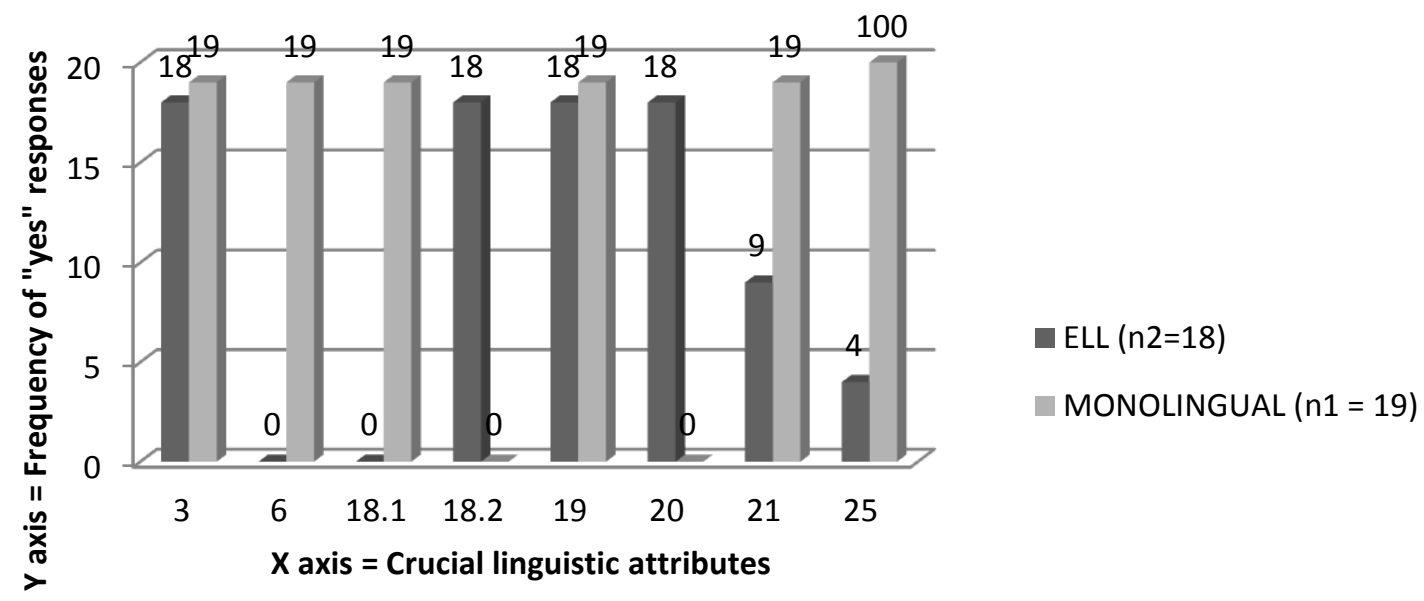

\section{FINDINGS OF THE QUALITATIVE STUDY}

The information in Table 1 indicates that all learners (both ELLs and English monolingual learners) included in the sample were born in Canada (Q3). The parents of English monolingual learners were born in Canada and have resided in Canada since birth. The parents of ELLs were not born in Canada. They immigrated to Canada as adults and have therefore not resided in Canada their entire lives. All the English monolingual learners speak English at home, while none of the ELLs speaks English at home (Q18.1). The home language of the ELLs, Mandarin or Cantonese, is spoken at home (Q18.2).

These findings on the biographical attributes indicate that the ELL and English monolingual learner groups differ with regard to parental residency in Canada and the language spoken at home. Once the significance of the differences between the two groups regarding HCD is validated and discussed in relation to the quantitative study, the profile established in the qualitative study can be used to describe an at-risk educational minority within the British Columbian education system. 


\section{THE QUANTITATIVE STUDY}

The quantitative research, as the second aspect of the complementary mixed-model design type, introduces objective numeric measurement into the investigation. In this instance, a comparative analysis methodology was followed which explored the relationship between reading, comprehension and vocabulary levels in ELLs and English monolingual learners. The variables used in the study include reading (decoding), comprehension and vocabulary. The strategy for comparative analysis followed in the study assisted in investigating the relationship between measurements of the literacy phenomenon between two sampled groups of the target population. The two sampled groups of the target population were represented by ELLs and English monolingual learners. The above relationships are indicated in terms of the significant differences that are reflected in group means (for the two sampled groups) for literacy measures calculated.

The following two quantitative assessment instruments apply to data information and competencies. These instruments were administered to both the ELL (experimental group) and English monolingual learners (comparison group).

(a) The Neale Analysis of Reading Ability (NARA) -- the aim of this analysis is an investigation into the reading and comprehension abilities of both the comparison group and the experimental group for comparative purposes. The passages were graded and learners were tested for rate, accuracy and comprehension. The diagnostic component of the NARA was applied in this comparative study as it investigates reading skills and comprehension.

(b) The Peabody Picture Vocabulary Assessment - the (PPVT-IV) instrument evaluates comprehension of the spoken word in Standard English and is thus a measure of the learner's vocabulary acquisition. It is a norm-referenced, untimed instrument that is individually administered. The examiner presents a series of pages, each page containing four coloured pictures. The learner selects the picture that best illustrates the meaning of the stimulus word, and it takes approximately 15 minutes to complete the assessment. The primary reason for the administration of this assessment is to ascertain the receptive vocabulary levels of both the comparison group and the experimental group in an attempt to relate a vocabulary deficit to the HCD. As indicated earlier in this article, vocabulary is strongly related to reading comprehension ability, since the learner must understand the meaning of the words in order to grasp the meaning of the text (Wagner, Muse, \& Tannenbaum, 2007:288).

\section{FINDINGS OF THE QUANTITATIVE STUDY}

The NARA and the PPVT-IV were administered to all respondents included in the sample. The aim of the NARA was to evaluate reading skills and comprehension, while the PPVT-IV evaluates comprehension of the spoken word in Standard English and is thus a measure of the learner's receptive vocabulary acquisition.

NARA

The 'uniform' standardised scores for each respondent were calculated as the raw scores divided by the standard deviation. Results of the $t$-test for independent samples, calculated on the standardised reading and comprehension scores are presented in Table 2. The test was done to determine whether the English monolingual learners differ significantly from the 
ELLs with regard to their reading comprehension assessment means, as measured on the NARA comprehension assessment.

Table 2: The $t$-test results on standardised reading and comprehension scores

\section{Table 2}

The $t$-test results on standardised reading and comprehension scores and correlation results between reading and comprehension for small independent samples with unequal variances (NARA) assessment standardised scores)

\begin{tabular}{|c|c|c|}
\hline \multicolumn{3}{|c|}{$t$-test on standardised comprehension scores } \\
\hline Statistics & Monolinguals & ELL \\
\hline $\begin{array}{l}\text { Mean }(\text { Stand/C) illustrated } \\
\text { in Box Plot }\end{array}$ & 104.4 & 92.55 \\
\hline Std Deviation (Stand/C) & 4.9127 & 7.679 \\
\hline $\begin{array}{l}t \text {-test statistic and } \\
\text { probability }\end{array}$ & \multicolumn{2}{|c|}{ Probability $(t$-statistic $=5.54)<0.0001 * * *$} \\
\hline \multicolumn{3}{|c|}{$t$-test on standardised reading scores } \\
\hline Statistics & Monolinguals & ELL \\
\hline $\begin{array}{l}\text { Mean (Stand/C) illustrated } \\
\text { in box plot }\end{array}$ & 106.21 & 108.78 \\
\hline Std deviation (Stand/C) & 4.10 & 7.29 \\
\hline $\begin{array}{l}t \text {-test statistic and } \\
\text { probability }\end{array}$ & \multicolumn{2}{|c|}{ Probability $(t$-statistic $=1.31)=0.20$} \\
\hline \multicolumn{3}{|c|}{ Correlation between comprehension and reading } \\
\hline $\mathbf{n}_{1}$ & 19 & 18 \\
\hline $\begin{array}{l}\text { correlation (r) } \\
\text { (Probability associated with } \\
\text { r) }\end{array}$ & $\begin{array}{l}0.83914 \\
(<0.0001)^{* * *}\end{array}$ & $\begin{array}{l}0.43009 \\
\text { (n.s.) }\end{array}$ \\
\hline
\end{tabular}

\section{Comprehension}

$t$-test for null hypothesis: $\mathrm{mu}_{1}-\mathrm{mu}_{2}=0$

\& alternative hypothesis: $\mathbf{m u}_{\mathbf{1}}>\mathbf{m u}_{\mathbf{2}}$

Difference $(\mathrm{D})=104.37$-- 92.556

$$
=11.81
$$

$\mathrm{S}_{1}=4.9127 ; \mathrm{S}_{2}=7.679$ Std diff 2.1

$t$-test statistic $=5.54$ (Satterthwaite

approximation) and

probability $\left(t_{\text {statistic }}=5.54\right)<0.0001^{* * * *}$

Significance legend:

?: $10 \%$ level of significance

*: $5 \%$ level of significance

$* *$ : $1 \%$ level of significance

$* * *: 0.1 \%$ level of significance

\section{$t$-test calculations:}

\section{Reading}

$t$-test for null hypothesis: $\mathbf{m u}_{1}-\mathbf{m u}_{2}=\mathbf{0}$

\& alternative hypothesis: $\mathbf{m u}_{\mathbf{1}}>\mathbf{m u}_{\mathbf{2}}$

Difference $(D)=108.78-106.21$

$$
=2.57
$$

Std diff $=1.96$

$t$-test statistic $=1.31$ (Satterthwaite approximation) and probability $\left(t_{\text {statistic }}=1.31\right)=0.20$

\section{FINDINGS}

The difference between the comprehension averages of the comparison group and the experimental group is highly significant at the $0.1 \%$ level of significance. Further distinctions 
between the two groups became evident in that a high correlation exists between the reading and comprehension ability of learners included in the comparison group, while reading and comprehension performance in the experimental group did not indicate a significant correlation.

\section{PPVT-IV}

This first indication of difference in comprehension between the ELL and the monolingual learner, as evident in the NARA, was further investigated by means of the PPVT-IV.

A $t$-test for independent samples of unequal variance was done to determine significant differences between the English monolingual learners and ESLs with regard to their receptive vocabulary as measured on the PPVT-IV. The results of the two-sample $t$-test for independent samples, of unequal size, are presented in Table 3 below.

Table 3: The $t$-test results for small independent samples with unequal variances

(PPVT-IV standardised vocabulary scores)

\begin{tabular}{|l|c|}
\hline \multicolumn{3}{|c|}{ Table 3 } \\
t-test results for small independent samples with unequal variance (PPVT standardised \\
vocabulary scores)
\end{tabular}

\section{FINDINGS}

A summary of the results of the $t$-test analysis presented in Table 3 indicates that the difference between the average scores of the comparison group (English monolingual learners) and the experimental group (ELL) for receptive vocabulary measured by means of the PPVT-IV is highly significant $(<0.001)$.

The aforementioned results focused on the findings of the research data collected for the purpose of isolating and specifying an at-risk educational minority through the identification of the HCD. 


\section{CONCLUSION}

One of the most astonishing feats of the young learner is the ability to acquire language. With regard to spoken language development, the early years are highly representative of this notable feat. As the learner enters school, he or she is required to use the newly developed language skills which have been accumulating over the past 5 to 6 years, as instruments to further learning, in other words, reading and writing. Classrooms today are filled with learners with varying degrees of 'word poverty' (Moats, 2001:8) or language deficit, with disparate causes, and each learner has unique needs.

However, what happens to these limited language proficient (LLP) learners as they embark on their academic futures has serious consequences for the rest of their lives. Hart and Risley (2003) referred to this bleak reality and its serious implications as 'The early catastrophe': a catastrophe for the learners, their peers, the classroom, school, curriculum and finally society, where, with the resultant poor academic outcomes, the learners' futures are compromised and at risk.

The critical importance of language skills, both written and spoken, infers that differences and deficits determine the degree of the risk involved in the academic competence and ultimate academic outcomes of the ELL (Miller, 2004:12). Considering Vertovek's (2006:1) aptly named 'super diversity', culturally and linguistically diverse learners in 'immigrant-receiving countries' are often 'concentrated in settings of educational disadvantage when bilingualism and cultural background can be quickly transformed into explanations of poor performance' (Windle, 2004:12). It is therefore critical for teachers, who seek to 'empower' such learners, to be able to identify the nature of the academic challenges and deficits confronting them (Windle, 2004:95).

It is evident that fresh and novel ways of understanding and responding to the complex interplays of today's ELL acquiring first-time literacy in a SL need attention, if we are to move beyond a framework derived from an earlier and significantly different educational paradigm. Mitchell (2009:20) equated inclusive education with a 'Trojan Horse, in that it challenges education systems and schools to transform, re-align and re-engineer their missions, structure, curricula and pedagogies'.

The information obtained from the literature review and the findings of the empirical investigation responded to the research question and allowed us to conclude that an at-risk educational minority exists, identified by its cultural and linguistic differences and deficits and the presence of an HCD. This phenomenon 'makes slow growth of vocabulary in the early years far more ominous than it appears when viewed as one unfortunate phenomenon' (Wolf, 2007:103). Wolf summarises the essence of this study in her statement 'Reading never just happens. Not a word, sound, concept, or a social routine is wasted in the 2,000 days' that prepare the young learner for literacy acquisition: 'It is there from the start - or not -- with consequences for the rest of the children's literacy development, and for the rest of their lives' (Wolf, 2007:107). Further research is required, founded on deep empathy and a moral obligation to these young learners and their teachers, as they face their futures. As Mitchell (2009:20) pointed out, 'Inclusive education [not only] reflects the ethos of the education system and the culture in which it is embedded, but it also contributes to its shaping'. 
Dunn (1993: 31) captured the premise of this study when he stated, 'The way of words, of knowing and loving words, is a way to the essence of things, and to the essence of knowing.' Without the knowing of words, knowing in the sense of comprehending the written word (or for that matter, the spoken word) the essence or understanding of our world is diluted. Knowing the profile of the at-risk educational minority and their attendant HCD is to understand how to address this particular phenomenon in our global world. The most vulnerable, our children, are at risk.

1. End note: GIEN SNELGAR, E.C. 2010. Semiotic anomalies in English, as second language learners of immigrant parents acquire first time literacy. Unpublished MEd dissertation. Pretoria. University of South Africa.

\section{ACKNOWLEDGEMENT}

We thank Mrs Helene Muller, Senior Research Support Consultant in the research directorate at the University of South Africa for her statistical input and expertise. 


\section{REFERENCES}

BARNHILL, G. 2001. What is Asperger's syndrome? Intervention in school and clinic, 36 (5):259-265.

CAIN, K. \& J OAKHILL. 2007. Children's comprehension problems in oral and written language: A cognitive perspective. New York, NY: The Guilford Press.

GARNET, B \& C. AMAN. 2008. Academic performance of immigrant youth in Vancouver, Montreal and Toronto: The Vancouver context. Presented at the $10^{\text {th }}$ National Metropolis Conference in Halifax, Nova Scotia on April 4, 2008.

GIEN SNELGAR, EC. 2010. Semiotic anomalies in English, as second language learners of immigrant parents acquire first time literacy. Unpublished MEd dissertation. University of South Africa, Pretoria, RSA..

GOSWAMI, U. 2003. Early phonological development and the acquisition of literacy. In: Neuman, SB \& DK Dickenson (Eds.). Handbook of literacy research. New York, NY: The Guilford Press.

GUNDERSON, L. 2007. English only instruction in secondary schools: A critical examination. Mahwah, NJ: Lawrence Erlbaum.

HAKUTA, K. \& D AUGUST. 2001. Improving schooling for language-minority children: A research agenda of the National Council Institute of Medicine. Washington, DC: National Academy Press.

HART, B \& T RISLEY. 2003.The early catastrophe. American Educator, 27(4):6-9.

HUMAN EARLY LEARNING PARTENRSHIP (HELP) 2005. The British Columbia atlas of child development. Accessed on 12/11/2008. Available at:

http://www.earlylearning.ubc.ca/wpuploads/web.help.ubc.ca/2010/02/BCAAtlasofChi ldDevelopmentCD22-01-06

KANBAR, R. 2002. Economics, social science and development. World Development, 30 (3):477-486

KARMILOFF, K \& A KARMILOFF-SMITH. 2001. Pathways to language: From foetus to adolescent. Cambridge, MA: Harvard University Press.

MILLER, J, P KOSTOGIZ \& M GEARON.. 2009. Culturally and linguistically diverse classrooms. New dilemmas for teachers: New Perspectives on language education. Bristol, UK: Multilingual Matters.

MITCHELL, R \& F MYLES. 2004. Second language learning theories $\left(2^{\text {nd }}\right.$ ed.). London, UK: Hodder Education.

MITCHELL, D. 2009 (Ed.). Contextualizing inclusive education: Evaluating old and new international perspectives. New York, NY: Routledge.

MORROW, LM. 2001. Literacy development in young children: Research to practice. In: Goldbeck, L. (Ed.). Creating environments for learning in the early years: From research to practice. New Brunswick, NJ: Rutgers Graduate School of Education.

PROTOCOL FOR AGREEMENTS FOR MINORITY-LANGUAGE EDUCATION AND SECOND - LANGUAGE INSTRUCTION 2009-2010 to 2012-2013 between the Government of Canada and the Council of Ministers of Education, Canada. Accessed on 20/02/ 2009. Available at 
http://www.cmec.ca/Publications/Lists/Publications/Attachments/211/protocol-foragreements-2009-2013.pdf

ROBERTSON, HJ. 2005. Under the rainbow. Phi Delta Kappan. 86 (9):713-715.

ROBINSON, P \& P TROWER. 1988. Social skills training In Breckwell, GM, H Foot \& R Gilmour (Eds.). Doing school psychology: Laboratory and field exercises. ) New York, NY: Cambridge University Press: 172-184.

STATISTICS CANADA. 2006a. Immigration in Canada: A portrait of the foreign born population. Statistics Canada Catalogue no. 97-557-XIE: 5 Accessed on: 29/06/2008. Available at http://www40.statscan.ca/101/cst/demo11c.htm

STATISTICS CANADA. 2006b. The evolving linguistic portrait, 2006 census: Sharp increase in population with a mother tongue other than English or French. Accessed on 07/08/2011. Available at http://www12.statcan.ca/census-recensement/2006/assa/97-555/p2-eng.cfm

VORTOVEK, S. 2006. The emergence of super diversity. Centre on Migration Policy and Society. Working Paper no. 25, University of Oxford wp-06-25. Oxford, UK.

WAGNER, RK., AE. MUSE, \& KR. TANNENBAUM, 2007. Vocabulary acquisition: Implications for reading acquisition. London, UK: The Guildford Press.

WINDLE, J. 2004. Schooling, symbolism and social power: The hijab in Republican France. Australian Researcher 31(1):95--112.

WOLF, M. 2007. Proust and the squid. New York, NY: Harper Collins.

\section{BIOGRAPHICAL NOTE}

Norma Margaret Nel is an associate professor in the Department of Psychology of Education at the University of South Africa. Her responsibilities include post-graduate supervision. She has published articles in journals and books during her academic career of nine years at Unisa. Her fields of interest are Inclusive Education, Barriers to Learning, English Second Language and Learner Support.

Email address: nelnm@unisa.ac.za

Elizabeth Claire Gien Snelgar is the ESL family programme co-ordinator for the Salt Spring Literacy Society. She is currently a student at the University of South Africa. 